\title{
Difficult to Open or Remove Packaging Material
}

National Cancer Institute

\section{Source}

National Cancer Institute. Difficult to Open or Remove Packaging Material. NCI

Thesaurus. Code C63231.

Problem associated with difficulty for users to operate the device, specifically as it relates to the opening or removal of the outer wrapping. 